\title{
Determination of field strengths in the quiet Sun
}

\author{
A. López Ariste ${ }^{1}$, M. J. Martínez González ${ }^{2}$, and J. C. Ramírez Vélez ${ }^{3}$ \\ 1 THEMIS, CNRS UPS 853, C/ vía Láctea s/n, 38200 La Laguna, Tenerife, Spain \\ e-mail: arturo@themis.iac.es \\ 2 Instituto de Astrofísica de Canarias, C/ vía Láctea s/n, 38200 La Laguna, Tenerife, Spain \\ ${ }^{3}$ LESIA, Observatoire de Meudon, 92195 Meudon, France
}

Received 11 May 2006 / Accepted 1 December 2006

\begin{abstract}
Context. The magnetism in the quiet regions of the solar photosphere carries information on the dynamo processes and its interaction with the convection of the outer layers of the sun. Unfortunately, the scales of the magnetic structures on these regions are mostly unresolved. It is therefore instrumental to tell apart the intrinsic field strengths in those regions from the flux through the resolution element. This disentanglement has been far from obvious, leading to opposing views of the magnetic topology in the unresolved structures of the quiet Sun.

Aims. Our study contributes to the disentanglement of field strength from flux in the quiet Sun, at least, through the use of new observational constrains in the form of spectropolarimetry of Mn I lines observed in the solar spectrum.

Methods. The chosen Mn lines present a strong coupling with hyperfine structure resulting in spectral features, present or absent as a function of field strength alone. We observe one of those lines simultaneously and co-spatially with the Fe I lines at $630 \mathrm{~nm}$, at the core of the previous measurements.

Results. The inversion of the observed Fe lines results in either strong or weak fields depending on the initializations of the inversion algorithm. All the solutions show nevertheless equally good values for the $\sigma$ parameter and are therefore equally valuable as solutions. The Mn however selects unambiguously strong or weak fields, sometimes agreeing with the inversions of the Fe lines, but half the time disagreeing with them.

Conclusions. The Fe I lines at $630 \mathrm{~nm}$, in the conditions found in the quiet Sun, carry no binding information on field strength. A proper analysis of quiet Sun magnetism should necessarily pass through its simultaneous and co-spatial observation with other lines imposing constraints on field strength, as the Mn I lines here analyzed. Ultimately, the magnetic topology of the quiet Sun shall arise from the coherent analysis of all these lines, sensitive to the Zeeman effect.
\end{abstract}

Key words. Sun: photosphere - Sun: magnetic fields - polarization

\section{Introduction}

It has long been assumed that the photospheric network, the most readily accessible region of the quiet Sun may be made of small concentrations of $\mathrm{kG}$ fields, at least in the main magnetic cores (Muller 1983). However there is a long-standing discussion about the distribution of field strengths in the internetwork, where the signals have only recently been at reach of spectropolarimetric instruments. The controversy may be broadly described as follows: while the Fe I lines at $630 \mathrm{~nm}$ appear to result in strong $\mathrm{kG}$ field strengths (Sánchez Almeida \& Lites 2000; Domínguez Cerdeña et al. 2003; Socas-Navarro \& Lites 2004), the near-IR pair of lines of Fe I at $1.56 \mu \mathrm{m}$ shows a preponderance of field strengths in the range of the equipartition fields at 300-500 G (Lin 1995; Lin \& Rimmele 1999; Khomenko et al. 2003). The large effective Landé factors of those lines ( $g_{\text {eff }}=3$ and 1.45) and the long wavelength at which lines are found (the Zeeman splitting divided by the Doppler broadening being proportional to wavelength) would make the field strength measurements through them trustable, although perhaps more sensitive to the weaker component of the field distribution in the resolution element (Socas-Navarro \& Sánchez Almeida 2003). On the other hand, the readiness of the pair of Fe I lines at $630 \mathrm{~nm}$ and their strong polarimetric signals have resulted in successful and long-tested inversion codes that can cope with the full line formation in a magnetized plasma. The lines are also formed in similar enough conditions that one is tempted to just use line-ratio techniques (Howard \& Stenflo 1972; Stenflo 1973) to infer field strengths (Domínguez Cerdeña et al. 2003; Socas-Navarro et al. 2004). Both analysis, and in this work we shall confirm it, result in a predominance of $\mathrm{kG}$ fields. Nevertheless the particularities of the observation of the Stokes profiles are such (very low signals, very weak magnetic fluxes, mostly absence of any signal in Stokes $Q$ and $U$, small filling factors for measurable magnetic fields) that they do not allow the use of inversion codes straightforwardly and many authors have consequently restrained from doing it (e.g. Lites 2002).

Recently there has been some numerical work (Martínez González et al. 2006a,b; Collados 2006) giving good reason to those works and showing that, in those conditions the Fe lines at $630 \mathrm{~nm}$ mostly lack of any information on the field strength. The result of any inversion process will be therefore unconstrained by the observables but will rather depend on the implicit biases of the inversion algorithm. Most of those algorithms tend to fix in one way or another temperatures first, since the final profiles depend too strongly on temperature, a fact that often masks the response of the lines to the magnetic fields if left unchecked (Ruiz Cobo \& del Toro Iniesta 1992). The cited works show that invariably in quiet Sun conditions, once the thermodynamics fixed, the only path towards an acceptable fit goes through high 

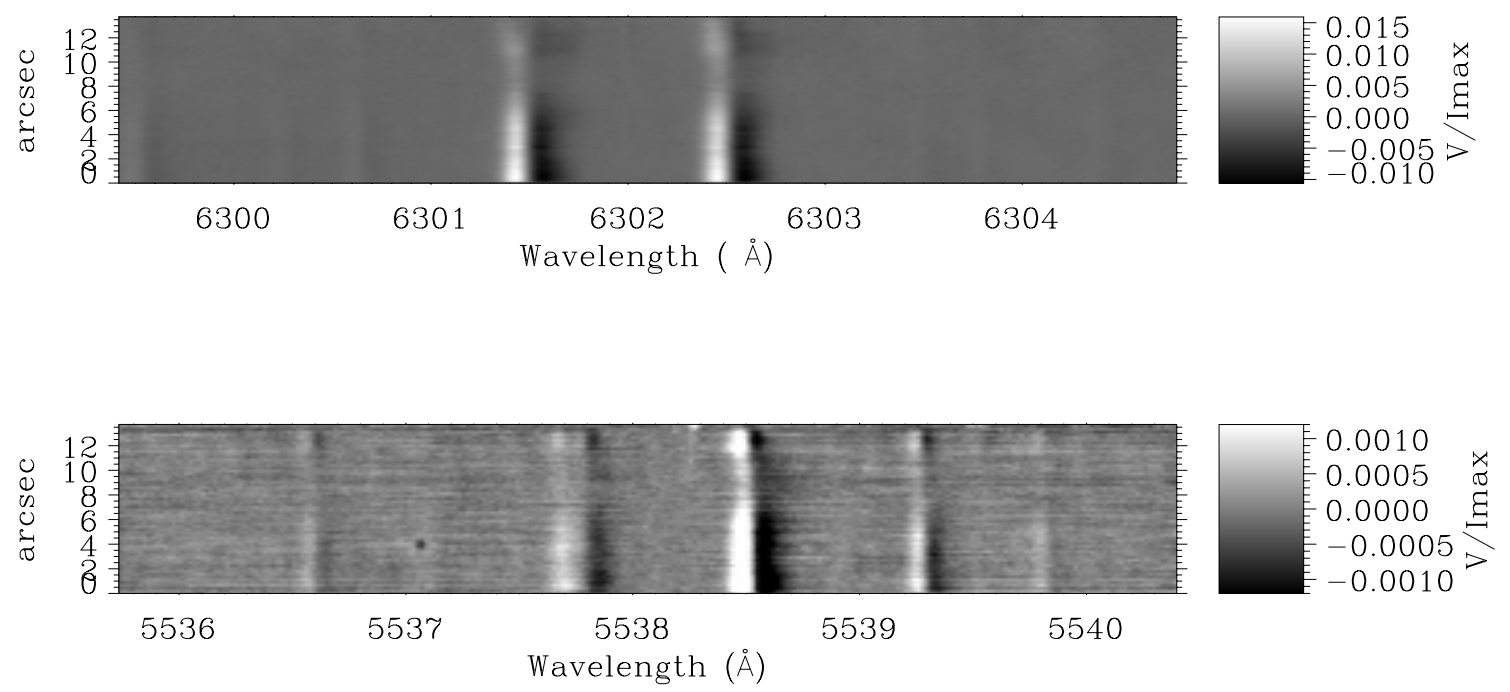

Fig. 1. Stokes $V$ images of the observed quiet Sun region at $630 \mathrm{~nm}$ region (top) including the two well-known Fe I lines, and the $553 \mathrm{~nm}$ region (bottom) with the studied $\mathrm{Mn}$ line at $553.77 \mathrm{~nm}$, as well as two neighboring Fe I lines.

field strengths. But conversely, if the magnetic field is forced to weak field strengths, the thermodynamic parameters adapt to that fact and identically satisfactory fits are produced with weak fields. Clear as it may appear, the number of cases that can be proposed for test is so big that it is difficult to be conclusive just on numerical tests.

To clarify this situation more observables are desirable. We found the Mn I line at $553.7 \mathrm{~nm}$ to be particularly suited to this since it produces a direct observable feature related to field strength like the near-IR lines, but it is placed at visible wavelengths and shares similar continuum and line formation process with the $\mathrm{Fe}$ I lines in the visible.

In the following sections, we should first handle the Fe lines data as usual, applying inversion codes and inferring field strengths. In Sect. 3 we shall compare the results from the inversion, and the Fe profiles themselves with the Mn line to see where do they agree and where they do not. The conclusion of the comparison, which will be discussed in the last section, confirms the numerical tests on these Fe I lines in the sense that they do not seem to carry binding information on the magnetic field strength. The Mn line results in field strengths which agree mostly with the near-IR general results in terms of field strengths.

\section{Observations}

On June 21st, 2005 we observed the quiet Sun at disc center with the THEMIS MTR mode (López Ariste et al. 2000) in 5 spectral domains simultaneously. The setup was such as to provide us with a noise level of $2 \times 10^{-4}$ the continuum intensity $\left(I_{\mathrm{c}}\right)$. This noise level allowed us to reach the weak magnetic signals in the photospheric internetwork, in particular for the Mn I line. We also observed simultaneously the pair of Fe I lines at $630 \mathrm{~nm}$. These lines produce stronger signals (almost one order of magnitude bigger than the Mn line for the same magnetic flux). The slow modulation cycle of the present THEMIS polarimeter results in a high-risk of seeing-induced crosstalk so we were forced to use a beam-exchange technique and correlation of signals in the continuum to discard any profile at risk of being polluted by crosstalk. This process came at a price in photons and, despite having collected enough photons per pixel to go beyond $10^{-5} I_{\mathrm{c}}$, we ended up with the stated $2 \times 10^{-4} I_{\mathrm{c}}$ noise level.

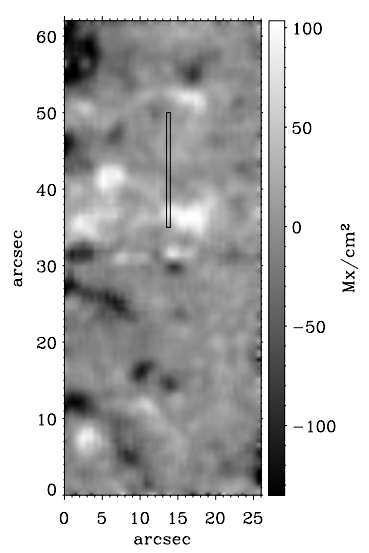

Fig. 2. Scan over the observed region shown in magnetograph signal. The framed subset region approximately corresponds to the region observed in the time series that provided the data for this work.

The data can be seen in Fig. 1. They consist of a long time series ( $5 \mathrm{~min}$, to be compared with granular lifetimes) with fixed slit position over a supergranule 10 arcsec to the north of disc center embedded in the region showed in Fig. 2, coming from a scan of the region made a few minutes later. The slit was 15.5 arcsec long and 0.5 arcsec wide. The image was sampled with 0.25 arcsec per pixel.

A second scan made a few minutes later serves the purpose of placing spatially the time series data in the supergranulation background (Fig. 2). This scan was made with low polarimetric sensitivity $\left(2 \times 10^{-3} I_{\mathrm{c}}\right)$ for the sake of speed. The region covered by the fixed slit in the time series can be seen to cover a patch of magnetic network in the bottom part and the intranetwork for the middle and upper parts of the slit. The predominance of the positive polarity in the whole supergranular cell can be ascertained from this scan as well.

\section{The inversion of $\mathrm{Fe}$ I $\lambda \lambda 630 \mathrm{~nm}$}

The LTE inversions of the data with the pair of Fe I lines at 630.1 and $630.2 \mathrm{~nm}$ are performed running the $\mathrm{SIR}^{1}$ code

\footnotetext{
${ }^{1}$ Stokes Inversion based on Response functions.
} 
(Ruiz Cobo \& del Toro Iniesta 1992). The physical parameters needed to compute the absorption matrix are specified in the model atmosphere which is discretized in the equally spaced logarithmic scale of the continuum optical depth at $500 \mathrm{~nm}$. At each grid point the temperature, microturbulence, line of sight velocity and the magnetic field vector are specified. The model atmosphere is completed with a set of depth-independent parameters: the macroturbulent velocity, the stray light contamination and the filling factor.

A two component scenario is assumed to reproduce the observed Stokes $I$ and $V$ profiles: a magnetic atmosphere covering some surface portion and a non magnetic one filling up the rest of the space. The lack of conspicuous linear polarization signals in the observed data allows us to set the magnetic field vector to be vertical in the inversions for the sake of simplicity. Some constraints are applied to the atmospheric physical quantities in order to obtain reliable data inversions. Magnetic field strength and microturbulent and line of sight velocities are supposed to be constant along the optical depth axis. The macroturbulent velocity is furthermore constrained to be the same for both atmospheres. Temperature gradients have enough degrees of freedom to independently cool or heat different atmospheric layers (5 nodes were left for the temperature so that it was able to reproduce thermal structures along the line of sight, the magnetic parameters had, on the other hand, just 1 node, i.e. they were constant). Under these conditions, we carried out two different inversion schemes. In the first one, we fixed the temperature to be the same both in the magnetic and in the non magnetic components. In the second one, we left them vary individually. Both procedures were initialized with a filling factor of $2 \%$ and a magnetic field strength of $500 \mathrm{G}$.

The magnetic field strengths recovered from the two previous inversion schemes are plotted in Fig. 3 as crosses and diamonds. Both evidence the presence of ubiquitous $\mathrm{kG}$ field strengths at quiet Sun regions, having slightly higher values when fixing the temperature gradients for both components.

From the work by Martínez González et al. (2006a), one also concludes that since the lines do not have information on the magnetic field strength, solutions with weak magnetic fields should also be a valid solution to the inverse problem. To test this, we repeated the inversion, this time with a very weak field $(300 \mathrm{G})$ as initial condition. Also, we fixed this value until the code had almost converged, and released the magnetic field strength as a free parameter to finish the convergence. The temperature stratification was kept as free parameters and independent in both components. The results are shown in Fig. 3 (dots). In this plot one can directly compare the retrieved field strengths with those of the previous inversion, also with temperatures free to vary in both atmospheres (crosses). The most important fact is that both solutions are completely valid as can be seen in Fig. 3 . The standard deviations $(\sigma)$ of the difference between the observed and fitted profiles in both inversion procedures are plotted one vs. the other. They turn out to be equal. This is further illustrated in the particular example shown in Fig. 4. The two inversions led to $1221 \mathrm{G}$ and $300 \mathrm{G}$ respectively, but the solution profiles are identical. And while the magnetic field strength greatly differs in the two inversions, the inferred magnetic flux is the same for both. The conclusion is that we cannot distinguish between $300 \mathrm{G}$ and $1200 \mathrm{G}$ in our data, but we can still trust the inferred magnetic flux. Whatever the magnetic field strength effects are on the profiles, they are compensated by small changes in the magnetic temperature gradient and a slightly higher magnetic microturbulent velocity.
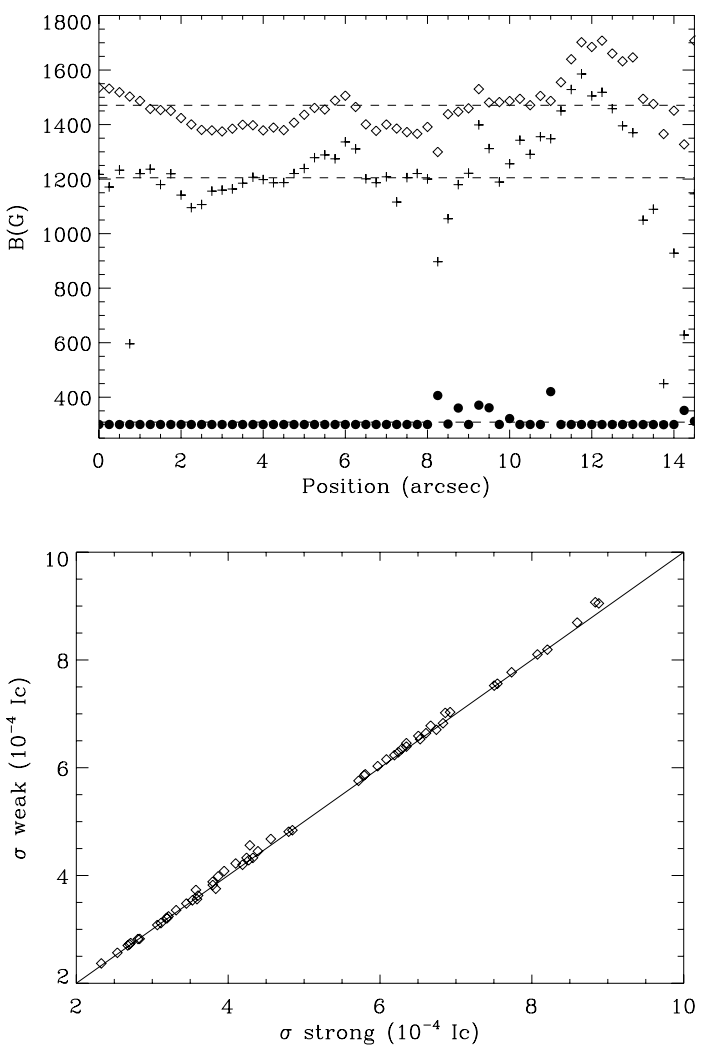

Fig. 3. Top panel: magnetic field strength retrieved from three different inversion routines: diamonds correspond to the inversion with fixed temperatures at the two components; crosses to the inversion with independent stratification temperatures both in the magnetic and in the non magnetic atmospheres and dots to the one with a $300 \mathrm{G}$ as initial condition for the magnetic field strength. Bottom panel: comparison of the standard deviations of the difference between the observed and fitted profiles in both "weak" (vertical axis) and "strong" (horizontal axis) inversion procedures. The overplotted solid line is the diagonal of the plot.

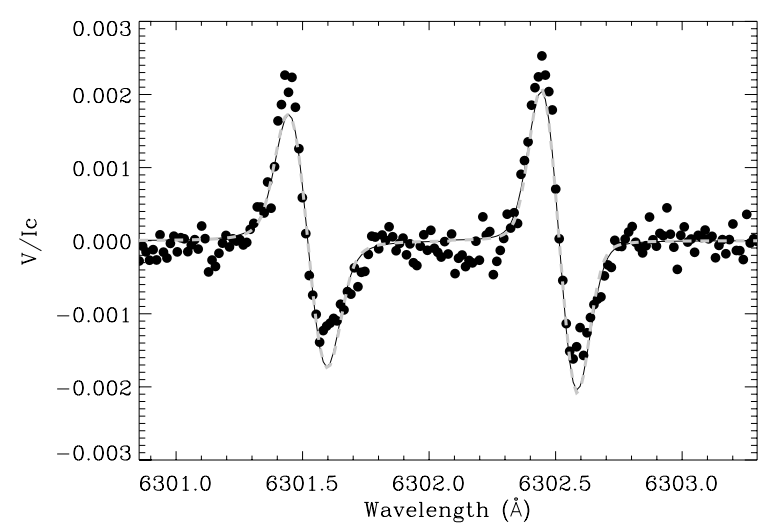

Fig. 4. Example of a particular observational profile (dots) and its two different inversions. The one initialized with a $300 \mathrm{G}$ field which lead to a magnetic field strength of $300 \mathrm{G}$ (grey dashed line) and the one initialized with $500 \mathrm{G}$ which lead to $1221 \mathrm{G}$ (solid black line).

\section{The comparison of $\mathrm{Fe} \mathrm{I} \lambda \lambda 630 \mathrm{~nm}$ and $\mathrm{Mn}$ I $\lambda \lambda 553.7 \mathrm{~nm}$}

The Mn I line at $553.7 \mathrm{~nm}$ was proposed by López Ariste et al. (2002) as an interesting line for magnetic field diagnostics because of its strong coupling between hyperfine structure and Zeeman effect. They showed in particular how the Stokes $V$ 

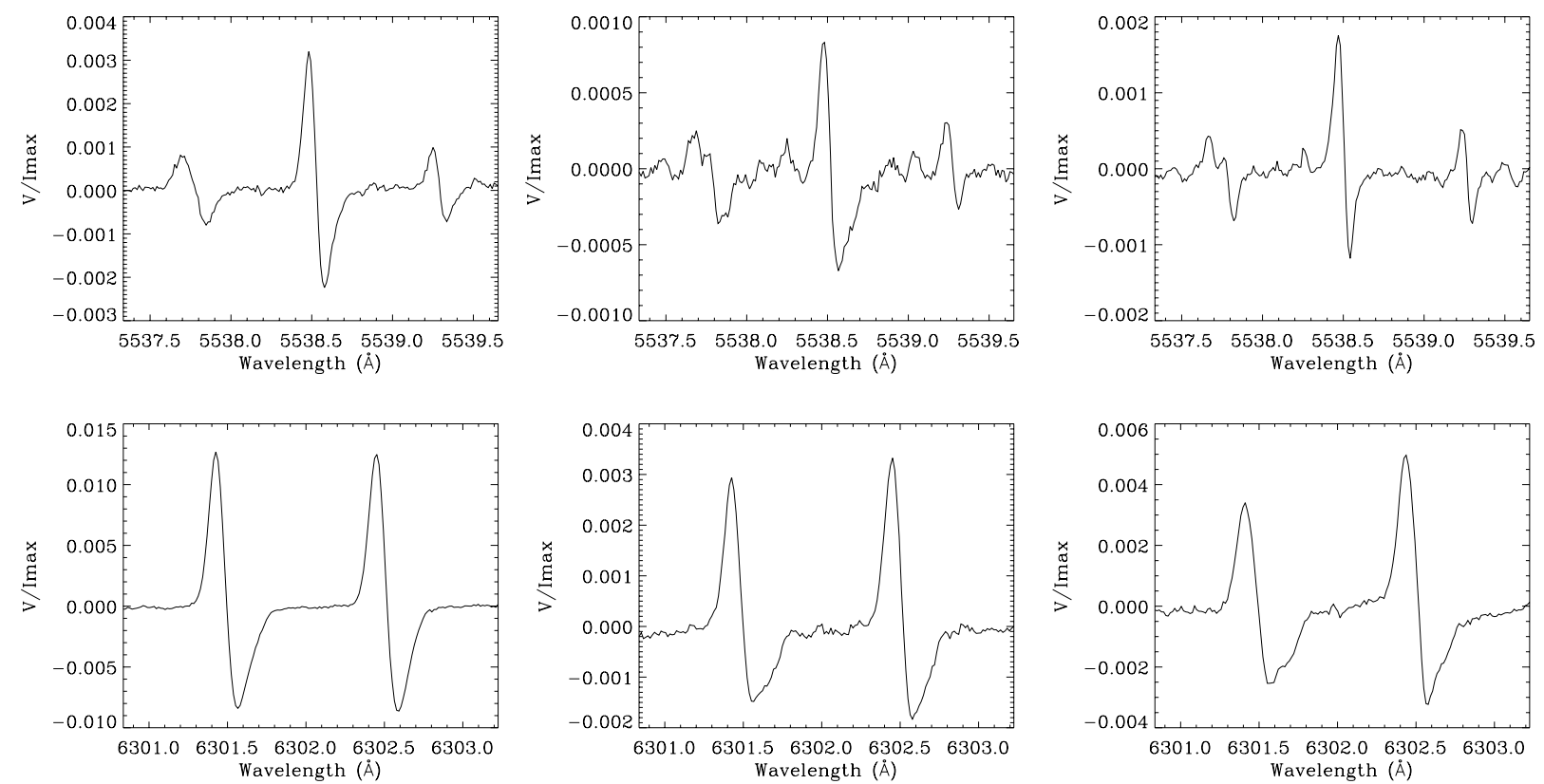

Fig. 5. Simultaneous and co-spatial profiles of Mn (top) and Fe (bottom) for different magnetic regimes, selected at three positions along the slit.

profile at fields below $600 \mathrm{G}$ shows an anomalous spectral feature between the two main Zeeman lobes. Such a feature disappears when the atom enters into Paschen-Back regime, thus recovering the profile its usual antisymmetric shape. Recently López Ariste et al. (2006) have observed that line in the photospheric network and internetwork and used the presence or absence of such a spectral feature to identify the predominance of intrinsically strong (above $800 \mathrm{G}$ ) or weak (below $600 \mathrm{G}$ ) fields in the observed regions. In a further step they proposed a toy model of a 2-component field topology, with one component at $1500 \mathrm{G}$ and a second one at $325 \mathrm{G}$, and used the ratio of the anomalous spectral feature amplitude to the main Zeeman lobe amplitude to infer the filling factors of both components. The result was that whenever the anomalous feature was present the strong field occupied surfaces at most one order of magnitude smaller than the weak fields. We shall mostly rely on these arguments for our present comparison.

In Fig. 5 we have selected for illustration three cases from the observations and whose simultaneously-measured Fe profiles were inverted as shown in the previous section. We shall use the results of the inversions with unrestricted field strength presented in the previous sections, since they are representative of the results traditionally quoted in the literature for the field strengths of the quiet Sun. The profiles are co-spatial ${ }^{2}$ and an average has been made over four pixels to increase the $\mathrm{S} / \mathrm{N}$ ratio in the benefit of the weaker Mn line. The leftmost profiles correspond to the bottom 1.25 arcsec of the slit. The Fe I lines at $630 \mathrm{~nm}$ are fully saturated with an amplitude ratio very close to 1 . The Mn I line shows a clear antisymmetric shape corresponding to an atom in the Paschen-Back regime and therefore above $800 \mathrm{G}$ of intrinsic field strength in rough agreement with the value of $1200 \mathrm{G}$ from the inversion of the Fe lines. The measured magnetic flux (see Fig. 7) falls in the range of 50 to $90 \mathrm{Mx} / \mathrm{cm}^{2}$ depending on what line of $\mathrm{Fe}$ is considered for the

\footnotetext{
${ }^{2}$ Over continuum images made in both spectral regions simultaneously the final co-spatiality has been measured at better than 1 pixel or 0.25 arcsec, for an average seeing slightly better than 1 arcsec.
}

measurement ${ }^{3}$. Such fluxes correspond to the photospheric network, where with widespread acknowledgment, strong kG fields are to be expected.

The rightmost profiles show the opposite case. They are picked from the upper part of the slit (13 through 15 arcsec). The Fe lines fall to a ratio of Stokes $V$ amplitudes of 0.6 and lower (see Fig. 6), similar to the ratio of effective Landé factors of the two lines, characteristic of the weak field regime. The inversion of those lines infers a field strength of 500 to $1000 \mathrm{G}$ depending on the model atmosphere. Simultaneously the Mn line, despite the lower $\mathrm{S} / \mathrm{N}$ ratio, shows a conspicuous anomalous spectral feature, confirmed in its nature by the narrower Zeeman lobes and the stepper slopes towards the zero crossing. The profile shape of the line is even more conspicuous when it is compared with the Fe I line at $553.93 \mathrm{~nm}$, which displays a similar $g_{\mathrm{eff}}$ and Stokes $V$ amplitude. Altogether the Mn line points towards a field strength well below the $600 \mathrm{G}$ threshold, and therefore confirming the weakest fields retrieved in some of the inversion schemes, though not the strongest ones. The fluxes fall in this case below $20 \mathrm{Mx} / \mathrm{cm}^{2}$, and this for both Fe lines, corresponding to an enhanced internetwork which is, nevertheless populated by a clear predominance of fields below $600 \mathrm{G}$. The low spatial resolution of the image smears the conclusion, but the fact that this is a magnetically-enhanced internetwork with nevertheless intrinsically weak fields is difficult to arrange with the presence of $\mathrm{kG}$ fields of zero average over our resolution element. If any, the weaker fields are more easily affected by the presence of random polarities, and not the strong and hypothetically more buoyant $\mathrm{kG}$ fields. We conclude therefore that the absence of $\mathrm{kG}$ fields in these profiles is not just a coincidence.

The agreement among lines fully disappears in the case of the middle profiles of Fig. 5. They have been picked around

\footnotetext{
3 To get this estimate on the magnetic flux, we have made use of the magnetograph formula on the Fe I line at $630.25 \mathrm{~nm}$ but also on the Fe I line at $553.85 \mathrm{~nm}$. This latter line lies close to the $\mathrm{Mn}$ line and is made of a perfect blend of two transitions. The resulting effective Landé factor was unknown, but we measure it, for low fluxes, to be 1.25. For high fluxes it appears to differ from this value and the two measurements differ.
} 

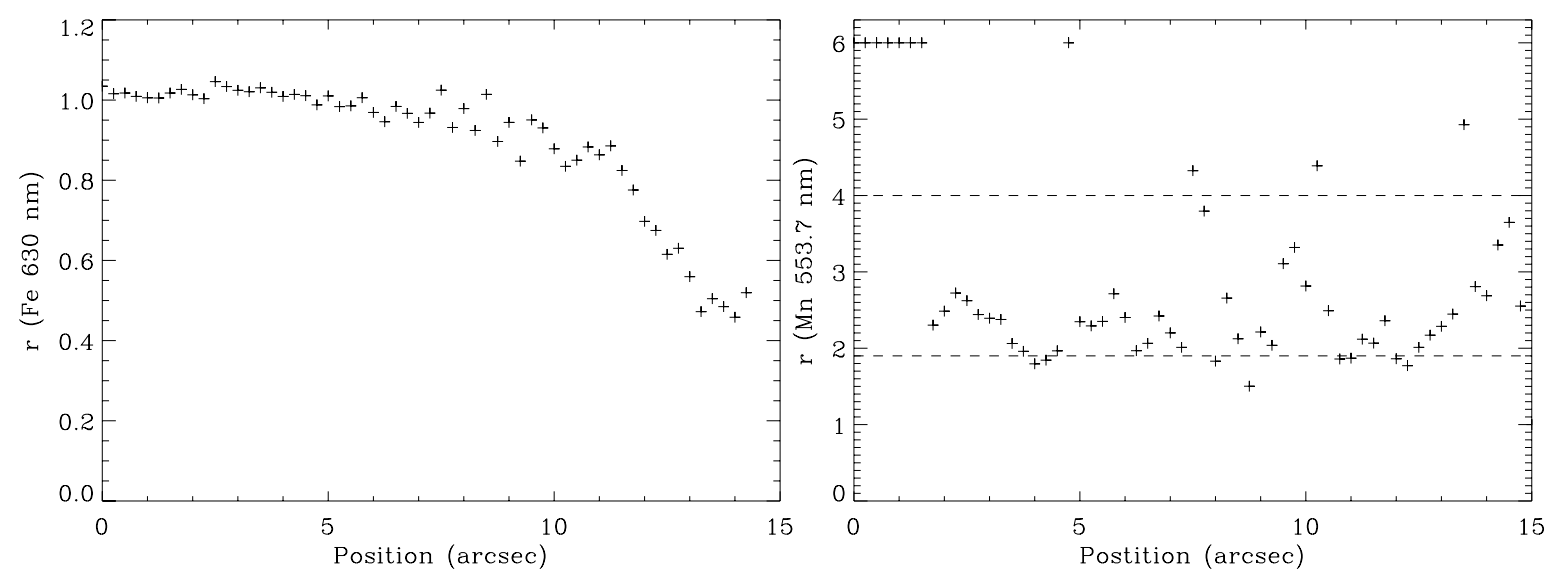

Fig. 6. Left: ratio of amplitudes of the Stokes $V$ Zeeman lobes in the pair of Fe I lines at $630 \mathrm{~nm}$ for all points along the slit. Right: ratio of the Zeeman lobe to the hyperfine spectral feature amplitudes in the Mn line. The dashed horizontal lines mark the minimum value of the ratio from simulations and the maximum value for weak fields (atom not in Paschen-Back regime). Whenever the profiles do not show the hyperfine feature the ratio has been set at 6 .

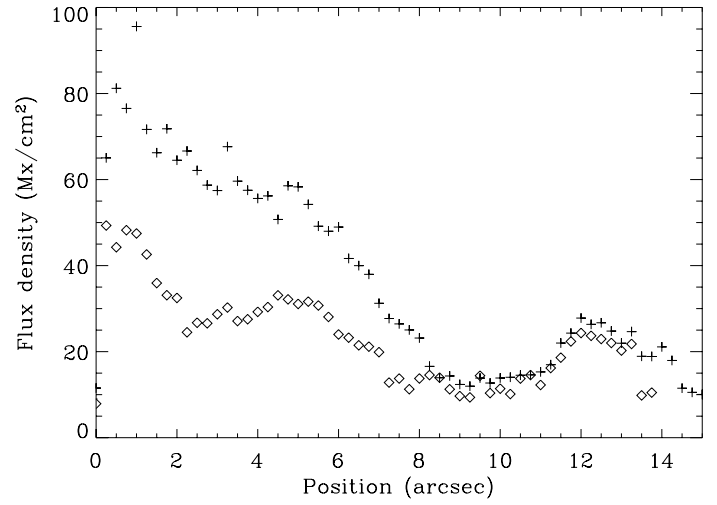

Fig. 7. Longitudinal magnetic flux density measured on the Fe I lines at $630.25 \mathrm{~nm}$ (crosses) and $553.85 \mathrm{~nm}$ (diamonds), for all points along the slit. The Fe I line at 553.85, close to the studied Mn line, is made of a perfect blend of two Fe transitions, whose contributions to the effective Landé factor were unknown. A value of 1.25 appears as a good match for the weak flux regions. At higher fluxes the effective Landé factor appears to differ from this value.

the point at 10 arcsec. The even lower $\mathrm{S} / \mathrm{N}$ makes identification trickier, but from the full slit image in Fig. 1 it should be obvious that the anomalous spectral feature placed at the correct wavelength and with acceptable amplitudes is not the result of any residual fringing or noise, something which is the origin of similar amplitude features observed on the continuum wavelengths. The narrower Zeeman lobes and the stepper slopes once more come to support the identification of the anomalous feature, which is finally confirmed by the inversions presented at the end of this section. Once more the $\mathrm{Mn}$ line points toward fields below 600 G. Simultaneously the Fe lines show an amplitude ratio around 0.9 corresponding to the saturation regime and traditionally identified with strong fields. All the inversion schemes produce field strengths of $1200 \mathrm{G}$, in full agreement with the ratio of amplitudes, and in full disagreement with the Mn line. The flux density places these profiles again in a magneticallyenhanced internetwork region.

More generally, we can compare, for all points along the slit, the field strength produced by the inversion of the Fe line at $630 \mathrm{~nm}$ with the ratio of amplitudes of the Zeeman lobe and the hyperfine spectral feature. In Fig. 6 the latter ratio is plotted for those points where it can be detected. Those points for which the profiles show a normal antisymmetric shape are given an ad-hoc ratio of 6 . The dashed lines in the plot show the lower and upper limits of the ratio for an $\mathrm{Mn}$ atom in the weak field regime. The lower limit is actually set by the atomic structure of the Mn atom and the particular geometry of the observations, while the upper limit marks the boundary of the passing into the Paschen-Back regime and, therefore, the kG fields. Next to this plot we produce a plot of the ratio of Stokes V amplitudes in both Fe I lines at $630 \mathrm{~nm}$. This ratio has traditionally been considered a proxy of the field strength (e.g. Socas-Navarro et al. 2004). In the weak field regime two lines identical except for its effective Landé factor should show a ratio corresponding to the ratio of their effective Landé factors. The Fe I lines at $630 \mathrm{~nm}$ are not strictly identical, but a ratio of amplitudes of 0.6 is traditionally considered to be proof of weak fields. Such a ratio is found near the top of the slit (12 arcsec and higher) coinciding with the lower field strengths retrieved by the inversion code in the scheme where temperatures are set free and independent in the two atmospheres, and also coinciding with the Mn profiles producing a clear hyperfine feature, signaling fields below $600 \mathrm{G}$.

For points below 8 arcsec the ratio of the Fe lines ranges from 0.8 to 1 , and is traditionally considered as the result of magnetic saturation and strong fields. For the lower 2 arcsec the Mn agrees and produces an antisymmetric profile, signature of atoms in the Paschen-Back regime and, therefore, with field strengths above $800 \mathrm{G}$. But for the positions 2 through $10 \mathrm{arcsec}$, the Mn line shows up the hyperfine spectral feature with ratios corresponding to fields below $600 \mathrm{G}$, while the Fe lines continue to show field strengths of $\mathrm{kG}$ and ratios above 0.8 . Since we know from the previous section that the inversion algorithms can be forced to produce solutions for the Fe lines with weak fields while maintaining the $\sigma$ values, we should trust the constraints on the field strength provided by the Mn line. In conclusion, we can assert that the Fe lines are not carrying binding information on the intrinsic field strengths on these regions (covering 8 out of the 15.5 arcsec observed), mostly placed in the internetwork regions.

While we are confident on the significance of the ratio in the $\mathrm{Mn}$ line, it is obvious that eventually we should rely on true inversions of the $\mathrm{Mn}$ line. In Fig. 8 we show the very first inversions of the 3 profiles selected to illustrate the differences between the $\mathrm{Fe}$ and the $\mathrm{Mn}$ lines. The inversion code is a very simple one, based on PCA techniques (Rees et al. 2000; 

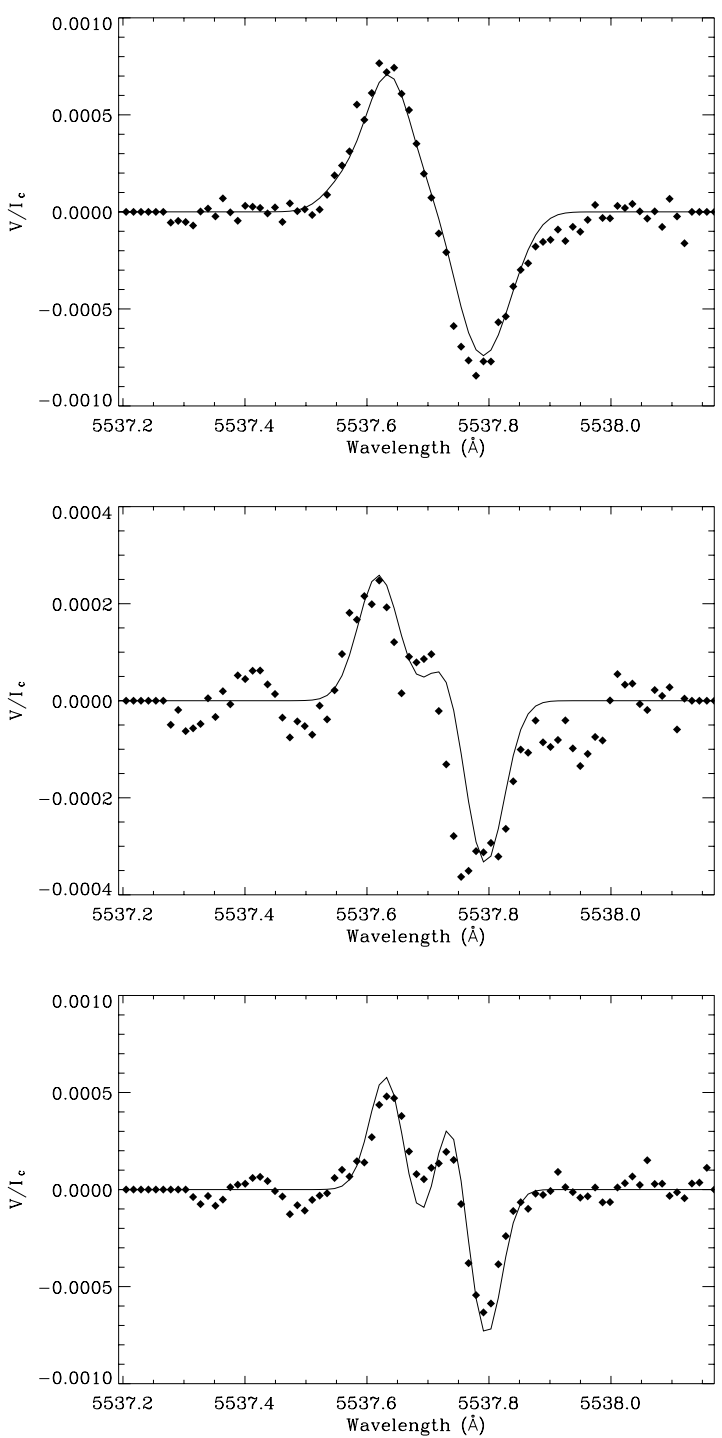

Fig. 8. Fits (continuous lines) to the observed Stokes $V$ profiles of the Mn line (circles) from the inversion for the tree cases selected for illustration in Fig. 6. The field strengths corresponding to the models fitting the observations are 1600,580 and $160 \mathrm{G}$ respectively from left to right.

López Ariste \& Casini 2002) and relying on a Milne-Eddington atmosphere. The description of the code and its performances is left for a future paper, but we found important to stress that solutions are found for the observed profiles and that the spectral feature on which we are basing our arguments is checked in position and amplitude by those solutions, and therefore confirmed in nature. The field strengths found by the inversion code (1600, 580 and $160 \mathrm{G}$ respectively) fully agree with our main conclusions.

\section{Conclusion}

We have analyzed simultaneous spectropolarimetric observations of the quiet Sun made with the traditional Fe I lines at $630 \mathrm{~nm}$ and the Mn I line at $553.7 \mathrm{~nm}$. The latter is characterized by its strong coupling with hyperfine structure and as a consequence, the Stokes $V$ profiles change shape with the intrinsic field strength. The field strength in the quiet sun has traditionally been inferred from the ratio of Stokes $V$ amplitudes in the pair of Fe lines (e.g. Socas-Navarro et al. 2004), as well as from inversion codes including temperature stratification
(Socas-Navarro \& Lites 2004). It is clearly shown now that the result of both methods (ratios and inversion algorithms) applied to real data is biased towards $\mathrm{kG}$ fields. The co-spatial and simultaneous Mn line shows field strengths that do not always agree with the Fe lines analysis, resulting in a much broader predominance of hG fields in the internetwork of the quiet Sun. The bias is confirmed when we produce inversion solutions with field strengths of $300 \mathrm{G}$, agreeing with the constrains imposed by the Mn line, and fitting the observed Fe lines as acceptably as the strong field ones. The identical values for the $\sigma$ confirm the goodness of both fits.

The Fe lines carry therefore no binding information on field strength, at least in the conditions set by the internetwork quiet Sun and our observations. One possibility is that the actual spatial resolution of the observations is too large compared to the scale of the magnetic features. In that case, since the longitudinal flux is correctly measured from the Fe lines, the strong field strength retrieved from the inversions of the Fe lines would just be compensated by a smaller filling fraction. One could therefore conclude that, by improving spatial resolution until the filling fraction approaches unity, the problem would be solved. However, the observed line-ratio values, at first order independent of the filling fraction, cannot be easily reproduced by a simple question of spatial resolution and filling factor. To our minds they point towards a fact also arising from the numerical simulations (Martínez González et al. 2006b): the thermodynamics of the Fe lines manage to get entangled with the field strength. We foresee that independently of the spatial resolution of the observations, it will be seen that the Fe lines at $630 \mathrm{~nm}$ carry no binding information on field strength. To retrieve this information one should rely therefore on lines in the near-IR or on lines like the Mn line that we analyzed in this work. The absence of information on field strength, does not mean that the $630 \mathrm{~nm}$ lines suddenly lack of any interest for solar magnetometry. It just means that our comprehension of the solar magnetism should necessarily pass through the simultaneous and co-spatial observation, and the subsequent coherent analysis, of the many lines in the solar spectrum sensitive to the magnetic field (Semel 1981).

\section{References}

Domínguez Cerdeña, I., Kneer, F., \& Sánchez Almeida, J. 2003, ApJ, 582, L55 Khomenko, E. V., Collados, M., Solanki, S. K., Lagg, A., \& Trujillo Bueno, J. 2003, A\&A, 408, 1115

Lin, H. 1995, ApJ, 446, 421

Lin, H., \& Rimmele, T. 1999, ApJ, 514, 448

Lites, B. W. 2002, ApJ, 573, 431

López Ariste, A., \& Casini, R. 2002, ApJ, 575, 529

Howard, R., \& Stenflo, J. O. 1972, Sol. Phys., 22, 402

López Ariste, A., Rayrole, J., \& Semel, M. 2000, A\&A, 142, 137

López Ariste, A., Tomczyk, S., \& Casini, R. 2002, ApJ, 580, 519

López Ariste, A., Tomczyk, S., \& Casini, R. 2006, A\&A, 454, 663

Martínez González, M. J., Collados, M., \& Ruiz Cobo, B. 2006a, A\&A, submitted

Martínez González, M. J., Collados, M., \& Ruiz Cobo, B. 2006b, Proceedings of the Solar Polarization Workshop 4, Boulder, Colorado, ed. R. Casini, \& B. W. Lites

Collados, M. 2006, Proceedings of the Solar Polarization Workshop 4. Boulder, Colorado, ed. R. Casini, \& B. W. Lites

Muller, R. 1983, Sol. Phys., 85, 113

Rees, D., López Ariste, A., Thatcher, J., \& Semel, M. 2000, A\&A, 355, 759

Ruiz Cobo, B., \& del Toro Iniesta, J. C. 1992, ApJ, 398, 375

Sánchez Almeida, J., \& Lites, B. W. 2000, ApJ, 532, 1215

Semel, M. 1981, A\&A, 97, 75

Socas-Navarro, H., \& Sánchez Almeida, J. 2003, ApJ, 593, 581

Socas-Navarro, H., \& Lites, B. W. 2004, ApJ, 616, 587

Socas-Navarro, H., Martínez Pillet, V., \& Lites, B. W. 2004, ApJ, 611, 1139

Stenflo, J. O. 1973, Sol. Phys., 32, 41 